\title{
ENTIRE FUNCTIONS AND MÜNTZ-SZÁSZ TYPE APPROXIMATION
}

\author{
BY \\ W. A. J. LUXEMBURG AND J. KOREVAAR( $\left.{ }^{1}\right)$
}

\begin{abstract}
Let $[a, b]$ be a bounded interval with $a \geqq 0$. Under what conditions on the sequence of exponents $\left\{\lambda_{n}\right\}$ can every function in $L^{p}[a, b]$ or $C[a, b]$ be approximated arbitrarily closely by linear combinations of powers $x^{\lambda_{n}}$ ? What is the distance between $x^{\lambda}$ and the closed span $S_{c}\left(x^{\lambda}\right)$ ? What is this closed span if not the whole space? Starting with the case of $L^{2}, C$. H. Müntz and O. Szász considered the first two questions for the interval [0, 1]. L. Schwartz, J. A. Clarkson and P. Erdös, and the second author answered the third question for $[0,1]$ and also considered the interval $[a, b]$. For the case of $[0,1]$, L. Schwartz (and, earlier, in a limited way, T. Carleman) successfully used methods of complex and functional analysis, but until now the case of $[a, b]$ had proved resistant to a direct approach of that kind. In the present paper complex analysis is used to obtain a simple direct treatment for the case of $[a, b]$. The crucial step is the construction of entire functions of exponential type which vanish at prescribed points not too close to the real axis and which, in a sense, are as small on both halves of the real axis as such functions can be. Under suitable conditions on the sequence of complex numbers $\left\{\lambda_{n}\right\}$, the construction leads readily to asymptotic lower bounds for the distances $d_{k}=d\left\{x^{\lambda_{k}}, S_{c}\left(x^{\lambda_{n}}, n \neq k\right)\right\}$. These bounds are used to determine $S_{c}\left(x^{\lambda} n\right)$ and to generalize a result for a boundary value problem for the heat equation obtained recently by V. J. Mizel and T. I. Seidman.
\end{abstract}

1. Introduction. Let $\left\{\lambda_{n}\right\}$ be a sequence of distinct complex numbers not too close to the imaginary axis: we require that, for some constant $\delta>0$ and all large $n$,

$$
\left|\operatorname{Re} \lambda_{n}\right| \geqq \delta\left|\lambda_{n}\right| \text {. }
$$

Problems of approximation by linear combinations of powers $x^{\lambda_{n}}$ (we will always take the principal values) on a bounded interval $[a, b]$ with $a>0$ led us to the following questions. How rapidly can an entire function $f(z)$ of exponential type $\tau>0$ tend to zero on both halves of the real axis, and what zeros (we are thinking

Received by the editors March 19, 1970.

AMS 1969 subject classifications. Primary 4130, 4218, 3057, 3058; Secondary 4225, 3565.

Key words and phrases. Müntz-Szász type approximation, approximation by powers $x^{\lambda}$, span of powers $x^{\lambda_{n}}$, closed span, approximation in $L^{p}[a, b]$, approximation in $C[a, b]$, asymptotic inequalities for distances, entire functions of exponential type, zeros, functions of exponential type, separation condition for zeros, infinite products, smallness, finite Fourier transforms, Fourier inversion, Paley-Wiener theorem, supporting interval, harmonic function, subharmonic function, Poisson integral, maximum principle, boundary value problems, heat equation.

( $\left.{ }^{1}\right)$ Work of first author supported in part by NSF grant GP-14133, that of the second author by NSF grant GP-8445. 
of the points $i \lambda_{n}$ ) can it have outside small angles containing those halves? Separate answers to the two questions can be found in the literature, but not in a form that makes them easily applicable to problems such as that of determining the distance in $L^{p}[a, b](1 \leqq p<\infty)$ between $x^{\lambda_{k}}$ and the closed span $S_{c}\left(x^{\lambda_{n}}, n \neq k\right)$ of the other powers $x^{\lambda}$. One has the following

THEOREM. Let $\tau>0$ be given, let $\omega(t)$ be a nonnegative increasing function defined for $t \geqq 0$, and let $\left\{\lambda_{n}\right\}$ be a sequence as above. Then there exists an entire function $f(z)$ of exponential type $\tau$ such that

$$
|f(x)| \leqq \exp \{-\omega(|x|)\}, \quad-\infty<x<\infty,
$$

and

$$
f\left(i \lambda_{n}\right)=0, \quad n=1,2, \ldots,
$$

if and only if both

$$
\int_{1}^{\infty} \frac{\omega(t)}{t^{2}} d t<\infty
$$

and

$$
\sum^{\prime} \frac{1}{\left|\lambda_{n}\right|}<\infty
$$

(Here and in the following, $\Sigma^{\prime}$ and $\Pi^{\prime}$ denote sums and products including all terms or factors of the form indicated with nonvanishing denominator.)

In view of (1.1), the necessity of conditions (1.4) and (1.5) follows readily from standard results (see $\$ 2$, and cf. [1, pp. 85, 86], [3, p. 38], [11, p. 28]) that are usually derived from T. Carleman's theorem [2]. In the other direction, several authors have noted the existence of "finite" Fourier transforms $f(x)$ not identically zero (hence, restrictions of entire functions of positive exponential type) which satisfy conditions of smallness closely related to (1.2), (1.4). They include R. E. A. C. Paley and N. Wiener [15, pp. 16, 24], A. E. Ingham [5], A. Wintner [18], [19], [20], [21], B. Jessen and A. Wintner [6], N. Levinson [10], [11, p. 81], T. Kawata [7], and S. Mandelbrojt [12]. However, only S. Mandelbrojt looked at these functions also as entire functions.

For given $\tau>0$ and given $\omega(t)$ and $\left\{\lambda_{n}\right\}$ such that (1.4) and (1.5) hold we construct an entire function $f(z)$ of exponential type $\tau$ satisfying (1.2) and (1.3) in the form

$$
f(z)=c z \prod^{\prime}\left(1+\frac{z^{2}}{\lambda_{n}^{2}}\right) \prod_{n=0}^{\infty} \cos \left(\varepsilon_{n} z\right) .
$$

Products of the second kind were introduced by A. Wintner (loc. cit.) for real $z=x$.

Using the above theorem we prove very simply that the sequence of powers $\left\{x^{\lambda} n\right\}$ spans one (and all) of the spaces $C[a, b]$ and $L^{p}[a, b](1 \leqq p<\infty)$ if and only if

$$
\sum^{\prime} \frac{1}{\left|\lambda_{n}\right|}=\infty
$$


For the sufficiency of (1.7) when $\operatorname{Re}\left|\lambda_{n}\right| \geqq \delta\left|\lambda_{n}\right|$ (cf. B. Ja. Levin [9, p. 416]); the more difficult part is to prove that (1.7) is necessary. For real $\lambda_{n}$ the complete result was obtained by L. Schwartz [16]; cf. also the closely related work by J. A. Clarkson and P. Erdös [4] and the second author [8]. The methods used by these authors would also work for nonreal $\lambda_{n}$. However, they compare approximation on $[a, b]$ with approximation on $[0,1]$; our method is much more direct. Corresponding results for $C[0,1]$ and $L^{p}[0,1](1 \leqq p<\infty)$ are much older and due to C. H. Müntz [14] and O. Szász [17]; T. Carleman [2] was the first to give a complexanalysis proof for the interval $[0,1]$ (cf. also $[15$, p. 32]).

Suppose, finally, that the sequence $\left\{\lambda_{n}\right\}$ satisfies (1.1), (1.5) and the separation condition

$$
\left|\lambda_{m}-\lambda_{n}\right| \geqq|m-n| \rho
$$

where $\rho>0$. All we need of (1.8) is the conclusion that there exists a constant $A$ such that no disc in the plane contains more than $1+A R$ points $\lambda_{n}$ where $R$ is the radius of the disc. Under these conditions our construction gives immediately the following asymptotic inequalities for distances in $C[a, b]$ and $L^{p}[a, b]$ : for every $\varepsilon>0$,

$$
\begin{aligned}
d_{k}=d\left\{x^{\lambda_{k}}, S_{c}\left(x^{\lambda_{n}}, n \neq k\right)\right\} & \geqq(b-\varepsilon)^{\mathrm{Re} \lambda_{k}} \quad \text { as } \operatorname{Re} \lambda_{k} \rightarrow+\infty, \\
& \geqq(a+\varepsilon)^{\operatorname{Re} \lambda_{k}} \quad \text { as } \operatorname{Re} \lambda_{k} \rightarrow-\infty .
\end{aligned}
$$

These estimates can be used to determine the closed span $S_{c}\left(x^{\lambda_{n}}, n=1,2, \ldots\right)$ under conditions (1.1), (1.5) and (1.8) (cf. [16], [4] and [8] for the case of real $\lambda_{n}$ ). Recently, inequalities of the form (1.9) have proved useful in the discussion of certain interesting boundary-value problems (see the work of V. J. Mizel and T. I. Seidman [13] for the heat equation where $\lambda_{n}=n^{2}$, and cf. §8).

2. Necessity of (1.4) and (1.5). Let $f(z)$ be an entire function of exponential type $\tau$ such that (1.2) holds with a nonnegative increasing function $\omega(t)$. Then $|f(x)|$ is bounded by one on $(-\infty, \infty)$; hence for all $z=x+i y$ (cf. Boas [1, p. 82]),

$$
|f(z)|=|f(x+i y)| \leqq e^{\tau|y|} .
$$

LEMMA 2.1 (cf. [3, p. 38]). Let $f(z)$ as above satisfy (1.2) with a nonnegative increasing function $\omega(t)$ such that

$$
\int_{1}^{\infty} \frac{\omega(t)}{t^{2}} d t=\infty
$$

Then $f=0$.

Proof. We consider the auxiliary function

$$
g(z)=e^{i \tau z} f(z), \quad \operatorname{Im} z \geqq 0 .
$$

Clearly

$$
\log |g(z)| \leqq 0, \quad \log |g(x)| \leqq-\omega(|x|)
$$


We would like to conclude that the subharmonic function $\log |g(z)|$ is bounded by the Poisson integral of $-\omega(|t|)$. However, the latter integral diverges! To get around the difficulties we set

$$
\begin{aligned}
\omega_{p}(t) & =\omega(t) & & \text { for } 0 \leqq t<p, \\
& =0 & & \text { for } t>p,
\end{aligned}
$$

and we introduce the harmonic function

$$
u_{p}(x+i y)=\frac{1}{\pi} \int_{-\infty}^{\infty} \frac{y}{(x-t)^{2}+y^{2}}\left\{-\omega_{p}(|t|)\right\} d t, \quad y>0 .
$$

Suitably normalizing $\omega_{p}(t)$ at points of discontinuity we will have $u_{p}(x+i 0)$ $=-\omega_{p}(|x|)$; we will set $u_{p}(x)=u_{p}(x+i 0)$. Also $u_{p}(z) \rightarrow 0$ uniformly as $z \rightarrow \infty$ in the upper half-plane. Thus for every $\varepsilon>0, \log |g(z)| \leqq u_{p}(z)+\varepsilon$ both on the real axis and on large semicircles with center 0 ; by the maximum principle the inequality will hold throughout the upper half-plane. Letting $\varepsilon \downarrow 0$ one finds that

$$
\log |g(z)| \leqq u_{p}(z), \quad \operatorname{Im} z>0 .
$$

By (2.2) $u_{p}(z) \rightarrow-\infty$ as $p \rightarrow \infty$. Letting $p \rightarrow \infty$ in (2.5) one concludes that $\log |g(z)|=-\infty$. Thus $g(z)=0$ for $\operatorname{Im} z>0$, and hence $f=0$.

LEMMA 2.2 (cf. [11, p. 28]). Let $f(z)$ be an entire function satisfying (2.1), and suppose that its zeros $z_{1}, z_{2}, \ldots$ are such that

$$
\Sigma^{\prime}\left|\operatorname{Im} \frac{1}{z_{n}}\right|=\infty
$$

Then $f=0$.

Proof. Introduce $g(z)$ as in the beginning of the preceding proof. Letting $\Pi^{*}$ denote a product over the zeros $z_{n}$ in the upper half-plane, the function

$$
g(z) \prod_{n=1}^{p} * \frac{1-z / \bar{z}_{n}}{1-z / z_{n}}
$$

will also be analytic for $\operatorname{Im} z \geqq 0$ and bounded by 1 . It follows that for each $p$,

$$
|g(i y)| \leqq \prod_{n=1}^{p} *\left|\frac{1-i y / z_{n}}{1-i y / \bar{z}_{n}}\right|, \quad y>0
$$

Now for fixed $y$ and large $z_{n}$ in the upper half-plane,

Thus if

$$
\left|\frac{1-i y / z_{n}}{1-i y / \bar{z}_{n}}\right|<1-y\left|\operatorname{Im} \frac{1}{z_{n}}\right|
$$

$$
\sum_{n=1}^{\infty}\left|\operatorname{Im} \frac{1}{z_{n}}\right|=\infty
$$

then $g(i y)=0$ and hence $f=0$. Similarly for the zeros $z_{n}$ in the lower half-plane. 
COROLlary. For the zeros $w_{n}=i \lambda_{n}$ of $f(z)$ such that $\left|\operatorname{Im} w_{n}\right| \geqq \delta\left|w_{n}\right|$, one has

$$
\left|\operatorname{Im} \frac{1}{w_{n}}\right| \geqq \delta \frac{1}{\left|w_{n}\right|}=\delta \frac{1}{\left|\lambda_{n}\right|} \text {. }
$$

Thus if the series in (1.5) diverges so does the series (2.6) and hence $f=0$.

3. Construction of functions satisfying (1.2). It will be sufficient if we can construct a function $f(z)$ of exponential type less than a given number $\delta>0$ which satisfies (1.2) for all large $|x|$. Indeed, given $f^{\prime}(z)$ one can multiply it by a function $c \cos \mu z$ to obtain a function $f(z)$ of prescribed type $\tau>0$ which satisfies (1.2) for all $x$.

Using an idea of A. Wintner [20] we try for $\tilde{f}(z)$ a product of the form

$$
\prod_{n=1}^{\infty} \cos \left(\varepsilon_{n} z\right)
$$

Here we take $\varepsilon_{n} \downarrow 0$ and such that

$$
\sum_{1}^{\infty} \varepsilon_{n}<\delta
$$

Then the product will converge to an entire function $\tilde{f}(z)$ such that

$$
|\tilde{f}(x+i y)| \leqq \exp \left\{\left(\sum_{1}^{\infty} \varepsilon_{n}\right)|y|\right\},
$$

hence $\tilde{f}(z)$ will be of exponential type $<\delta$.

For real $x$ we will have the inequality

$$
|\tilde{f}(x)| \leqq(\cos \gamma)^{v(x)}
$$

where $\gamma$ is any given number between 0 and $\frac{1}{2} \pi$ and $\nu(x)$ is the number of positive integers $n$ such that $\left|\cos \left(\varepsilon_{n} x\right)\right| \leqq \cos \gamma$.

We will take $\gamma<1$ and $x>0$ ( $f$ is even), and count only those $n$ for which

$$
\gamma \leqq \varepsilon_{n} x \leqq 1 \text {. }
$$

Taking

$$
\cos \gamma=1 / e
$$

we want to make $\nu(x) \geqq \omega(x)$ as $x \rightarrow \infty$.

Condition (3.4) may be rewritten as

$$
x \leqq 1 / \varepsilon_{n} \leqq \rho x, \quad \rho=1 / \gamma .
$$

It will be convenient to represent $1 / \varepsilon_{n}$ as $\varphi(n)$ where $\varphi(y)$ is a positive increasing function defined for $y \geqq 0$. Introducing the inverse function $\psi$ of $\varphi$ we can then write (3.6) as $\psi(x) \leqq n \leqq \psi(\rho x)$. It follows that

$$
\nu(x) \geqq \psi(\rho x)-\psi(x),
$$


and we will assure that $\nu(x) \geqq \omega(x)$ by defining

$$
\psi(x)=\frac{1}{\log \rho} \int_{a}^{x} \frac{\omega(t)}{t} d t, \quad a>0 .
$$

We need a strictly increasing function $\psi(x)$, hence for (3.8) to work we have to assume that $\omega(t)$ is positive for $t>a$. This is no restriction: if $\omega(t)=0$ for all $t$ we could simply take $f(z)=1$. We will impose a further condition on $a$ later. Since $\omega(t)$ is increasing, (3.8) implies that

$$
\psi(\rho x)-\psi(x)=\frac{1}{\log \rho} \int_{x}^{\rho x} \frac{\omega(t)}{t} d t \geqq \omega(x), \quad x \geqq a .
$$

Hence by (3.3), (3.5) and (3.7),

$$
|\tilde{f}(x)| \leqq(\cos \gamma)^{v(x)} \leqq e^{-\omega(|x|)}, \quad|x| \geqq a .
$$

We still have to make sure that the series $\sum_{1}^{\infty} \varepsilon_{n}$ converges and has sum $<\delta$. Clearly

$$
\sum_{1}^{N} \varepsilon_{n}=\sum_{1}^{N} \frac{1}{\varphi(n)}<\int_{0}^{N} \frac{1}{\varphi(y)} d y=\int_{a}^{\varphi(N)} \frac{1}{x} d \psi(x)=\frac{1}{\log \rho} \int_{a}^{\varphi(N)} \frac{\omega(x)}{x^{2}} d x .
$$

Thus when the integral in (1.4) converges, so does the series $\sum_{1}^{\infty} \varepsilon_{n}$, and its sum will be bounded by $(1 / \log \rho) \int_{a}^{\infty}\left(\omega(x) / x^{2}\right) d x$. Taking $a$ sufficiently large we can guarantee (3.2).

Summarizing we have the following

THEOREM 3.1. Let $\tau$ be an arbitrary given number $>0$ and let $\omega(t)$ be any nonnegative increasing function such that (1.4) holds. Then there exists an entire function

$$
f(z)=c \cos \mu z \tilde{f}(z)=c \prod_{n=0}^{\infty} \cos \left(\varepsilon_{n} z\right)
$$

of exponential type $\tau$ which satisfies the inequality (1.2) everywhere on the real axis.

For related results, cf. [15, pp. 16, 24], [5], [18], [19], [20], [21], [6], [10], [11, p. 81], [7] and [12].

4. Functions which vanish on a given sequence. Let $\left\{w_{n}\right\}$ be an arbitrary finite or infinite sequence of complex numbers (not containing infinitely many naughts) such that

$$
\sum^{\prime} \frac{1}{\left|w_{n}\right|}<\infty
$$

it is no restriction to assume that $0=\left|w_{1}\right|=\cdots=\left|w_{m}\right|<\left|w_{m+1}\right| \leqq \cdots$. The simplest entire function that vanishes on the sequence $\left\{w_{n}\right\}$ is

$$
z^{m} \prod_{n>m}\left(1-\frac{z}{w_{n}}\right),
$$


but unless the $w_{n}$ are on or very close to the imaginary axis, this function is larger on the real axis than we want it to be. We therefore form the function

$$
F(z)=z^{m} \prod_{n>m}\left(1-\frac{z^{2}}{w_{n}^{2}}\right) ;
$$

we will investigate its growth.

For $t \geqq 0$, let

$$
n(t)=\sum_{0<\left|w_{n}\right| \leqq t} 1 .
$$

Observe that $n(t)=0$ for $t$ near 0 and that $n(t) / t \rightarrow 0$ as $t \rightarrow \infty$ (if there are infinitely many $w_{n},(4.1)$ implies that $\left.n /\left|w_{n}\right| \rightarrow 0\right)$. It follows that the integral

$$
\int_{0}^{\infty} \frac{n(t)}{t^{2}} d t \quad\left(=\int_{0}^{\infty} \frac{d n(t)}{t}=\sum_{n>m} \frac{1}{\left|w_{n}\right|}\right)
$$

converges.

We now estimate $F(z)$ :

$$
\begin{aligned}
\log |F(z)| & \leqq m \log ^{+}|z|+\sum_{n>m} \log \left(1+\frac{|z|^{2}}{\left|w_{n}\right|^{2}}\right) \\
& =m \log ^{+}|z|+\int_{0}^{\infty} \log \left(1+\frac{|z|^{2}}{u^{2}}\right) d n(u) \\
& =m \log ^{+}|z|+\int_{0}^{\infty} \frac{n(u)}{u} \frac{|z|^{2}}{|z|^{2}+u^{2}} d u
\end{aligned}
$$

Denoting the last member by $\theta(|z|)$, it is easy to see that $\theta(t) / t \rightarrow 0$ as $t \rightarrow \infty$, hence $F(z)$ is an entire function of exponential type 0 . More precisely, $\theta(t)$ is a nonnegative increasing function for $t \geqq 0$ such that

$$
\int_{0}^{\infty} \frac{\theta(t)}{t^{2}} d t \quad\left(=m+\frac{1}{2} \pi \int_{0}^{\infty} \frac{n(u)}{u^{2}} d u\right)<\infty
$$

THEOREM 4.1. Let $\left\{w_{n}\right\}$ be any sequence of complex numbers (not containing infinitely many naughts) that satisfies condition (4.1). Then formula (4.2) defines an entire function $F(z)$ of exponential type 0 such that the $w_{n}$ occur among its zeros, and for which

$$
|F(x)| \leqq \exp \{\theta(|x|)\}, \quad-\infty<x<\infty,
$$

with a nonnegative increasing function $\theta(t)$ satisfying (4.5).

5. The principal result. Combining Theorems 4.1 and 3.1 we obtain

THEOREM 5.1. Let $\tau$ be an arbitrary given positive number, let $\left\{w_{n}\right\}$ be any sequence of complex numbers (not containing infinitely many naughts) that satisfies condition (4.1), and let $\omega(t)$ be any increasing function defined for $t \geqq 0$ such that (1.4) holds. Let $F(z)$ be the function defined by (4.2), $\theta(t)$ the function defined just below (4.4). 
Let $f(z)$ be as in $\$ 3$, except that $f(z)$ should correspond not to the given $\omega(t)$ but to the admissible increasing function $\omega(t)+\theta(t)$. Then the product

$$
G(z)=F(z) f(z)
$$

is an entire function of exponential type $\tau$ such that the $w_{n}$ occur among its zeros while

$$
|G(x)| \leqq \exp \{-\omega(|x|)\}, \quad-\infty<x<\infty .
$$

Suppose now that $\exp \{-\omega(x)\}$ belongs to $L^{2}(0, \infty)$. Then $G(x)$ belongs to $L^{2}(-\infty, \infty)$, hence by the Paley-Wiener theorem ([15, p. 13], cf. [1, p. 103]) one can represent $G(z)$ as a Fourier transform

$$
G(z)=\int_{-\tau}^{\tau} g(t) e^{-i z t} d t
$$

with $g(t)$ in $L^{2}(-\tau, \tau)$. Since $|G(z)|$ is even, the supporting interval of $g(t)$ is the whole interval $[-\tau, \tau]$ (cf. [1, p. 108]). When $\exp \{-\omega(x)\}$ tends to zero faster than any power of $1 / x$ as $x \rightarrow \infty$ (as is the case when $\omega(x)=x^{1 / 2}$ ), the inverse Fourier transform $g(t)$ of $G(x)$ will be of class $C^{\infty}$ on $(-\infty, \infty)$. Multiplying $G(z)$ by a suitable exponential $e^{-i \sigma z}$ we thus obtain

THEOREM 5.2. Let $\left\{w_{n}\right\}$ be an arbitrary sequence of complex numbers (not containing infinitely many naughts) that satisfies condition (4.1), and let $[\alpha, \beta]$ be an arbitrary bounded interval. Then there exists a $C^{\infty}$ function $g(t)$ on $(-\infty, \infty)$ with supporting interval $[\alpha, \beta]$ whose Fourier transform

$$
G(z)=\int_{\alpha}^{\beta} g(t) e^{-i z t} d t
$$

has the form

$$
z^{m} \prod^{\prime}\left(1-\frac{z^{2}}{w_{n}^{2}}\right) \cdot c e^{-i \sigma z} \prod_{n=0}^{\infty} \cos \left(\varepsilon_{n} z\right)
$$

with $\varepsilon_{n}>0, \varepsilon_{n} \downarrow 0$ for $n \geqq 1, \sum_{0}^{\infty} \varepsilon_{n}=\tau<\infty, \sigma-\tau=\alpha, \sigma+\tau=\beta$.

6. Müntz-Szász type theorems for $[a, b]$. Lemma 2.2 and Theorem 5.2 enable us to give a relatively simple proof of

THEOREM 6.1. Let $[a, b]$ be a closed bounded interval such that $a>0$, and let $\left\{\lambda_{n}\right\}$ be a sequence of distinct complex numbers such that (1.1) holds for all large $n$. Then the sequence of powers $\left\{x^{\lambda}\right\}$ spans one (and all) of the spaces $C[a, b]$ and $L^{p}[a, b](1 \leqq p<\infty)$ if and only if $(1.7)$ is satisfied.

For $\operatorname{Re} \lambda_{n} \geqq \delta\left|\lambda_{n}\right|$ the sufficiency of (1.7) was noted by B. Ja. Levin [9]; for real $\lambda_{n}$ the theorem was proved by L. Schwartz [16].

Proof. (i) Suppose that (1.7) holds. It will be sufficient to show that the powers $x^{\lambda_{n}}$ span $C[a, b]$. Assuming they do not, there must be a measure $d \mu$ of total mass 1 on $[a, b]$ such that

$$
\int_{a}^{b} x^{\lambda_{n}} d \mu(x)=0, \quad n=1,2, \ldots
$$


We now introduce the function

$$
f(z)=\int_{a}^{b} x^{-i z} d \mu(x)=\int_{\alpha}^{\beta} e^{-i z t} d \mu\left(e^{t}\right)
$$

$\alpha=\log a, \beta=\log b$. Clearly $f(z)$ is entire and of exponential type, bounded by $\int_{a}^{b}|d \mu|=1$ for real $z$, and such that $f\left(i \lambda_{n}\right)=0$. However, summing over all sufficiently large $n$, so that $\lambda_{n} \neq 0$ and (1.1) is satisfied, and using (1.7),

$$
\sum\left|\operatorname{Im} \frac{1}{i \lambda_{n}}\right|=\sum\left|\operatorname{Re} \frac{1}{\lambda_{n}}\right| \geqq \delta \sum \frac{1}{\left|\lambda_{n}\right|}=\infty .
$$

Thus by Lemma 2.2, $f=0$ and hence $d \mu=0$, a contradiction.

(ii) Suppose now that (1.5) holds. In this case it will be sufficient to show that the powers $x^{\lambda_{n}}$ fail to span $L[a, b]$. That is, we want to prove the existence of a bounded integrable function $h(x)$ on $[a, b]$ different from the zero function and such that

$$
\int_{a}^{b} x^{\lambda} h(x) d x=0, \quad n=1,2, \ldots
$$

Setting $x=e^{t}$, equation (6.1) requires that the function

$$
G(z)=\int_{a}^{b} x^{-i z} h(x) d x=\int_{\alpha}^{\beta} e^{-i z t} h\left(e^{t}\right) e^{t} d t
$$

vanish on the sequence $\left\{i \lambda_{n}\right\}$. We can satisfy this condition by setting $h\left(e^{t}\right) e^{t}=g(t)$ with $g(t)$ as in Theorem 5.2, taking $w_{n}=i \lambda_{n}$.

REMARK. In part (ii) we have not used (1.1). A slight modification of part (ii) will show that under condition (1.5), no power $x^{\lambda_{k}}$ is in the closed span of the other powers $x^{\lambda_{n}}$. In fact, one need only replace $h(x)$ and $G(z)$ by $h_{k}(x)$ and $G_{k}(z)$ as defined in (7.3), (7.4). To obtain estimates for the distance $d_{k}$ between $x^{\lambda_{k}}$ and $S_{c}\left(x^{\lambda_{n}}, n \neq k\right)$ other than the trivial one, $d_{k}>0$, we have to impose further conditions on the sequence $\left\{\lambda_{n}\right\}$.

7. Inequalities. We will now prove the asymptotic estimates (1.9):

THeOREM 7.1. Let $[a, b]$ be a closed bounded interval such that $a>0$, let $\left\{\lambda_{n}\right\}$ be $a$ sequence of complex numbers satisfying (1.1) (for all large n), (1.5) and (1.8), and let $\varepsilon$ be any given positive number. Then the distances

$$
d_{k}=d\left\{x^{\lambda_{k}}, S_{c}\left(x^{\lambda_{n}}, n \neq k\right)\right\}
$$

in $C[a, b]$ and $L^{p}[a, b]$ satisfy the asymptotic inequalities (1.9).

Proof. It will be sufficient to consider the case of $L[a, b]$. Also, we need only consider the case where $\operatorname{Re} \lambda_{k} \rightarrow+\infty$; the other case will follow by the change of variable $x=1 / y$ in appropriate integrals for distances in $L[a, b]$.

For every bounded integrable function $h_{k}(x)$ such that

$$
\int_{a}^{b} x^{\lambda} h_{k}(x) d x=0, \quad n \neq k,
$$


one has

$$
\left|\int_{a}^{b} x^{\lambda} h_{k}(x) d x\right| \leqq d_{k} \sup \left|h_{k}(x)\right|
$$

We will take

$$
\int_{a}^{b} x^{-i z} h_{k}(x) d x=\int_{\alpha}^{\beta} e^{-i z t} h_{k}\left(e^{t}\right) e^{t} d t=G_{k}(z)
$$

where

$$
G_{k}(z)=z \prod^{(k)}\left(1+\frac{z^{2}}{\lambda_{n}^{2}}\right) e^{-i \sigma z} \prod_{n=0}^{\infty} \cos \left(\varepsilon_{n} z\right),
$$

with $\varepsilon_{n}>0, \sum_{0}^{\infty} \varepsilon_{n}=\tau, \sigma-\tau=\alpha=\log a, \sigma+\tau=\beta=\log b$. Here $\prod^{(k)}$ denotes a modified product over $n$ defined as follows: there is no factor with $\lambda_{n}=0$, the factor $1+z^{2} / \lambda_{k}^{2}$ is replaced by $1+z / i \lambda_{k}$, and if there is a positive integer $p=p_{k}$ such that $-\lambda_{p}$ is within distance $\frac{1}{2} \rho$ of $\lambda_{k}$, the factor $1+z^{2} / \lambda_{p}^{2}$ is replaced by $1-z / i \lambda_{p}$.

We observe that for all real $u$, and all $k$ so large that $\left|\lambda_{k}\right| \geqq 1+\frac{1}{2} \rho$ (so that for $\left.p=p_{k},\left|\lambda_{p}\right|>1\right)$,

$$
\begin{aligned}
\left|G_{k}(u)\right| & \leqq|u| \prod_{n \neq k, p}\left(1+\frac{u^{2}}{\left|\lambda_{n}\right|^{2}}\right)\left(1+\left|\frac{u}{\lambda_{k}}\right|\right)\left(1+\left|\frac{u}{\lambda_{p}}\right|\right) \prod_{n=0}^{\infty}\left|\cos \left(\varepsilon_{n} u\right)\right| \\
& \leqq|u|(1+|u|)^{2} \prod^{\prime}\left(1+\frac{u^{2}}{\left|\lambda_{n}\right|^{2}}\right) \prod_{n=0}^{\infty}\left|\cos \left(\varepsilon_{n} u\right)\right|=H(u),
\end{aligned}
$$

say. We will choose our numbers $\varepsilon_{n}$ such that $H(u)$ is integrable over $(-\infty, \infty)$ (cf. $\$ \S 4$ and 5). By Fourier inversion,

$$
h_{k}\left(e^{t}\right) e^{t}=\frac{1}{2 \pi} \int_{-\infty}^{\infty} G_{k}(u) e^{i t u} d u,
$$

hence by (7.5),

$$
\begin{aligned}
\sup _{a \leqq x \leqq b}\left|h_{k}(x)\right| & \leqq \frac{1}{a} \frac{1}{2 \pi} \int_{-\infty}^{\infty}\left|G_{k}(u)\right| d u \\
& \leqq \frac{1}{2 \pi a} \int_{-\infty}^{\infty} H(u) d u=M, \quad k \geqq k_{0} .
\end{aligned}
$$

By (7.2), (7.3) and (7.6),

$$
d_{k} \geqq(1 / M)\left|G_{k}\left(i \lambda_{k}\right)\right|, \quad k \geqq k_{0} .
$$

For $\operatorname{Re} \lambda_{k}>0$ we write

$$
\begin{aligned}
\left|G_{k}\left(i \lambda_{k}\right)\right| & =\left|\lambda_{k}\right| \prod^{(k)}\left|1-\frac{\lambda_{k}^{2}}{\lambda_{n}^{2}}\right| \exp \left(\sigma \operatorname{Re} \lambda_{k}\right) \prod_{n=0}^{\infty}\left|\cosh \varepsilon_{n} \lambda_{k}\right| \\
& =\left|\lambda_{k}\right| \prod^{(k)}\left|1-\frac{\lambda_{k}^{2}}{\lambda_{n}^{2}}\right| b^{\mathrm{Re} \lambda_{k}} \prod_{n=0}^{\infty} \frac{1}{2}\left|1+\exp \left(-2 \varepsilon_{n} \lambda_{k}\right)\right| .
\end{aligned}
$$

Thus the proof of (1.9) may be completed with the aid of the following lemmas. 
LEMMA 7.2. Under conditions (1.5) and (1.8),

$$
\left|\lambda_{k}\right| \prod^{(k)}\left|1-\frac{\lambda_{k}^{2}}{\lambda_{n}^{2}}\right| \geqq \exp \left(-\varepsilon\left|\lambda_{k}\right|\right) \quad \text { as }\left|\lambda_{k}\right| \rightarrow \infty
$$

LEMMA 7.3. Under conditions (1.1) (for large $n$ ) and (1.5),

$$
\prod_{n=0}^{\infty} \frac{1}{2}\left|1+\exp \left(-2 \varepsilon_{n} \lambda_{k}\right)\right| \geqq \exp \left(-\varepsilon\left|\lambda_{k}\right|\right) \text { as } \operatorname{Re} \lambda_{k} \rightarrow+\infty \text {. }
$$

Indeed, by (7.7), (7.8), the lemmas, and (1.1)

$$
\begin{aligned}
d_{k} & \geqq(1 / M) b^{\operatorname{Re} \lambda_{k}} \exp \left(-2 \varepsilon\left|\lambda_{k}\right|\right) \geqq(1 / M) b^{\operatorname{Re} \lambda_{k}} \exp \left(-(2 \varepsilon / \delta) \operatorname{Re} \lambda_{k}\right) \\
& \geqq\left(b-\varepsilon^{\prime}\right)^{\operatorname{Re} \lambda_{k}} \quad \text { as } \operatorname{Re} \lambda_{k} \rightarrow \infty .
\end{aligned}
$$

Proof of Lemma 7.2. (Cf. N. Levinson [11, p. 92]; Levinson proves a closely related inequality under the tacit assumption that all $\lambda_{n}$ lie in the right half-plane.)

(i) We begin by proving the corresponding inequality for

$$
\left|\lambda_{k}\right| \prod_{n \neq k}^{\prime}\left|1-\frac{\lambda_{k}}{\lambda_{n}}\right|
$$

To do this, we split the product into three parts: a first part where $\left|\lambda_{n}\right| \leqq \frac{1}{2}\left|\lambda_{k}\right|$, a second part where $\frac{1}{2}\left|\lambda_{k}\right|<\left|\lambda_{n}\right|<2\left|\lambda_{k}\right|$, and the third where $\left|\lambda_{n}\right| \geqq 2\left|\lambda_{k}\right|$. The factors in the first part have absolute value $\geqq 1$ and may be omitted. For those in the second part, of which there are $r$ with $n<k$ and $s$ with $n>k$, say, condition (1.8) gives

$$
\prod\left|\frac{\lambda_{n}-\lambda_{k}}{\lambda_{n}}\right| \geqq \prod \frac{(n-k) \rho}{2\left|\lambda_{k}\right|} \geqq r ! s !\left(\frac{\rho}{2\left|\lambda_{k}\right|}\right)^{r+s} \text {. }
$$

By (1.5), the sum $\sum 1 /\left|\lambda_{n}\right|$ over the $\lambda_{n}$ in (7.9), that is, the $\lambda_{n}$ such that $\frac{1}{2}\left|\lambda_{k}\right|<\left|\lambda_{n}\right|$ $<2\left|\lambda_{k}\right|$, must tend to zero as $k \rightarrow \infty$. Now this sum is bounded below by $(r+s) / 2\left|\lambda_{k}\right|$, hence $r$ and $s$ are of the form $\eta\left|\lambda_{k}\right|$ with small $\eta$ 's. It follows that for large $k$,

$$
\log r !+r \log \frac{1}{2} \rho-r \log \left|\lambda_{k}\right| \approx \eta\left|\lambda_{k}\right| \log (\eta \rho / 2 e) \geqq-\varepsilon\left|\lambda_{k}\right| ;
$$

similarly with $r$ replaced by $s$. Thus (7.9) has a lower bound of the right form. For the third part, finally,

$$
\log \prod\left|1-\frac{\lambda_{k}}{\lambda_{n}}\right| \geqq-2 \sum\left|\frac{\lambda_{k}}{\lambda_{n}}\right| \geqq-2\left|\lambda_{k}\right| \sum_{n>k} \frac{1}{\left|\lambda_{n}\right|} \geqq-\varepsilon\left|\lambda_{k}\right|
$$

as $k \rightarrow \infty$.

(ii) The case of the product in the lemma can be reduced to the above by arranging the points $\lambda_{n}$ (with $n \neq k$ ) and $-\lambda_{n}$ (with $n \neq p_{k}$ ) in a single sequence $\left\{\mu_{n}^{(k)}\right\}$ such that

$$
\left|\mu_{n}^{(k)}-\lambda_{k}\right| \geqq|n-k| \rho / 4 \text {. }
$$

To see that this is possible, observe that the open disc about $\lambda_{k}$ of radius $R$ contains at most $2 R / \rho$ points $\lambda_{n}$ (with $n \neq k$ ). The number of points $-\lambda_{n}$ in that disc is 
bounded (very crudely) by $1+4 R / \rho$; however, there is no point $-\lambda_{n}$ within distance $\frac{1}{2} \rho$ of the center. It follows that as $R$ increases through the value $\nu \rho / 4$, the number of points $\pm \lambda_{n}$ in the disc can increase to no more than $2 \nu, \nu=1,2, \ldots$. The result now follows.

Proof of Lemma 7.3. Since $\operatorname{Re} \lambda_{k} \rightarrow+\infty$, we may assume that $\operatorname{Re} \lambda_{k} \geqq \delta\left|\lambda_{k}\right|$. It is convenient to split the product into two parts: a first part where $2 \varepsilon_{n}\left|\lambda_{k}\right|>\frac{1}{2}$, and a second where $2 \varepsilon_{n}\left|\lambda_{k}\right| \leqq \frac{1}{2}$. For the factors of the first part, $\operatorname{Re} 2 \varepsilon_{n} \lambda_{k}>\frac{1}{2} \delta$, hence

$$
\frac{1}{2}\left|1+\exp \left(-2 \varepsilon_{n} \lambda_{k}\right)\right| \geqq \frac{1}{2}\left(1-\exp \left(-\operatorname{Re} 2 \varepsilon_{n} \lambda_{k}\right)\right)>\frac{1}{2}\left(1-e^{-\delta}\right) .
$$

Denoting the number of factors in the first part by $\nu$ one has $2 \varepsilon_{v}\left|\lambda_{k}\right|>\frac{1}{2}$; since $\varepsilon_{n} \downarrow 0$ for $n \geqq 1$ and $\sum \varepsilon_{n}$ converges, $\varepsilon_{v}$ has the form $\eta / \nu$ with small $\eta$. It follows that $\nu<4 \eta\left|\lambda_{k}\right|$; thus the first part has a lower bound of the right form. For $|z| \leqq \frac{1}{2}$,

$$
\log \frac{1}{2}\left|1+e^{-z}\right| \geqq \log \left(1-\frac{2}{3}|z|\right) \geqq-|z| .
$$

Thus the logarithm of the second part is bounded below by $-2\left|\lambda_{k}\right| \sum_{n>v} \varepsilon_{n}$, and the proof of Lemma 7.3 is finished.

\section{Applications. We begin by proving}

LEMMA 8.1. Let $p(x)$ be a finite sum of the form $\sum a_{n} x^{\lambda_{n}}$. Then in each of the spaces $C[a, b]$ and $L^{p}[a, b]$ (with $\left.a \geqq 0,1 \leqq p<\infty\right)$

$$
\left|a_{k}\right| \leqq \frac{1}{d_{k}}\|p(x)\|, \quad k=1,2, \ldots,
$$

where $d_{k}$ is the distance between $x^{\lambda_{k}}$ and the closed span of the other powers $x^{\lambda_{n}}$. The inequality holds also when $p(x)$ is the sum of an infinite series $\sum a_{n} x^{\lambda_{n}}$ which converges in the space considered.

Proof. Indeed,

$$
\|p(x)\|=\left|a_{k}\right|\left\|x^{\lambda_{k}}-\sum_{n \neq k} \frac{-a_{n}}{a_{k}} x^{\lambda_{n}}\right\| \geqq\left|a_{k}\right| d_{k} .
$$

As a first application we indicate how to determine the closed span

$$
S_{c}\left(x^{\lambda}, n=1,2, \ldots\right)
$$

in the spaces $C[a, b]$ and $L^{p}[a, b]$ (with $a>0$ ) under conditions (1.1), (1.5) and (1.8). Suppose $f(x)$ is a limit of finite linear combinations $p_{j}(x)=\sum a_{n j} x^{\lambda_{n}}$. Then by (8.1) the numbers $a_{k j}, j=1,2, \ldots$, form a Cauchy sequence, hence a convergent sequence; we denote its limit by $b_{k}$. Since the norms $\left\|p_{j}\right\|$ tend to $\|f\|$ they are bounded by a constant $M$. Thus by (8.1) and (1.9), given $\varepsilon>0$ there is a constant $A$ such that

$$
\begin{aligned}
\left|a_{k j}\right|,\left|b_{k}\right| & \leqq M(b-\varepsilon)^{-\operatorname{Re} \lambda_{k}} \cdot \text { when } \operatorname{Re} \lambda_{k}>A, \\
& \leqq M(a+\varepsilon)^{-\operatorname{Re} \lambda_{k}} \quad \text { when } \operatorname{Re} \lambda_{k}<-A .
\end{aligned}
$$


It follows from (8.2) that the series $\sum_{1}^{\infty} b_{n} x^{\lambda_{n}}$ is absolutely and uniformly convergent on every closed subinterval $\left[a^{\prime}, b^{\prime}\right]$ of $(a, b)$, and that its sum is the uniform limit of the $p_{j}(x)$ on such intervals. However, the $p_{j}(x)$ converge to $f(x)$ in $C[a, b]$ or $L^{p}[a, b]$. Hence

$$
f(x)=\sum_{1}^{\infty} b_{n} x^{\lambda_{n}}, \quad a<x<b
$$

(in an almost everywhere sense in the case of $L^{p}$ ).

In the other direction one can show that every function $f(x)$ of the form (8.3) which can be extended to a function in $C[a, b]$, or which is in $L^{p}[a, b]$, is in $S_{c}\left(x^{\lambda_{n}}, n=1,2, \ldots\right)$. Indeed, writing $f=f^{+}+f^{-}$, where $f^{+}$is the sum of the terms with $\operatorname{Re} \lambda_{n}>0$, one may apply to $f^{+}$a result of the second author [8, Theorem 2 , p. 751], and similarly for $f^{-}$.

One thus obtains

THEOREM 8.2. Let $[a, b]$ be a closed bounded interval such that $a>0$, and let $\left\{\lambda_{n}\right\}$ be a sequence of complex numbers satisfying (1.1) (for large $n$ ), (1.5) and (1.8). Then the closed span $S_{c}\left(x^{\lambda_{n}}, n=1,2, \ldots\right)$ in $C[a, b]$ or $L^{p}[a, b]$ is the subspace consisting of those functions $f(x)$ which for $a<x<b$ have a convergent representation (8.3).

For the special case of real $\lambda_{n}$, L. Schwartz [16] proved this result and more. Cf. also [4] and [8].

We finally apply the inequalities (1.9) to a situation which occurs in certain boundary-value problems. Let $S$ be any bounded set of positive measure in $R^{p}$, and let $\left\{\varphi_{n}(x)\right\}$ be a sequence of functions on $S$ which are continuous at a point $x_{0}$ of $S$ (often a boundary point) and such that for some constant $B$,

$$
\left|\varphi_{n}(x)\right| \leqq B\left|\varphi_{n}\left(x_{0}\right)\right|, \quad x \in S, \quad n=1,2, \ldots
$$

Now let $u(x, t)$ be a function given by a convergent series

$$
u(x, t)=\sum_{1}^{\infty} c_{n} \varphi_{n}(x) \exp \left(-\lambda_{n} t\right), \quad x \in S, \quad t>0 .
$$

Here the numbers $\lambda_{n}$ are assumed to satisfy conditions (1.5) and (1.8); instead of (1.1) we require that, for all large $n$,

$$
\operatorname{Re} \lambda_{n} \geqq \delta\left|\lambda_{n}\right| \text {. }
$$

Under the given conditions, the series (8.5) will converge absolutely and uniformly for $x \in S, t \geqq \varepsilon>0$.

The problem is as follows. Do the values $u\left(x_{0}, t\right)$ on some finite $t$-interval, $0<t<1$, say, uniquely determine the function $u(x, 1)$ on $S$, and does $u(x, 1)$ depend continuously on $u\left(x_{0}, t\right)$ ? In the special case of the heat equation considered by V. J. Mizel and T. I. Seidman [13], the problem takes the following 
interesting form (we take $p=1$ ). Does the temperature variation over the time interval $0<t<1$ at one end of an insulated rod determine the temperature distribution along the rod at time $t=1$, and does the latter depend continuously on the former? In this case one may take $S=[0, \pi], \varphi_{n}(x)=\cos n x, x_{0}=0$ and $\lambda_{n}=n^{2}$.

THEOREM 8.3. The function $u(x, 1)$ on $S$ is uniquely determined by $u\left(x_{0}, t\right), 0<t<1$, and depends continuously on it: if \|\|$^{\prime},\|\|^{\prime \prime}$ denote supremum or $L^{p}(1 \leqq p<\infty)$ norms on $[0,1]$ and on $S$, respectively, there is a constant $M$ such that $\|u(x, 1)\|^{\prime \prime}$ $\leqq M\left\|u\left(x_{0}, t\right)\right\|^{\prime}$.

Proof. We may take \|\|$^{\prime}$ to be the $L^{1}$ norm, \|\|$^{\prime \prime}$ to be the supremum norm. Let $0<\varepsilon<\frac{1}{2}$. For $t>0$,

$$
u\left(x_{0}, t\right)=\sum_{1}^{\infty} c_{n} \varphi_{n}\left(x_{0}\right) \exp \left(-\lambda_{n} t\right)
$$

because of (8.6) the series converges uniformly for $\varepsilon \leqq t \leqq 1$. Thus by (8.1), taking $a=e^{-1}, b=e^{-\varepsilon}$,

$$
\begin{aligned}
\left|c_{k} \varphi_{k}\left(x_{0}\right)\right| & \leqq \frac{1}{d_{k}} \int_{\exp (-1)}^{\exp (-\varepsilon)}\left|u\left(x_{0}, \log (1 / s)\right)\right| d s \\
& =\frac{1}{d_{k}} \int_{\varepsilon}^{1}\left|u\left(x_{0}, t\right)\right| e^{-t} d t \leqq \frac{1}{d_{k}}\left\|u\left(x_{0}, t\right)\right\|^{\prime} .
\end{aligned}
$$

Since $d_{k}>0$ it follows that the numbers $c_{k} \varphi_{k}\left(x_{0}\right)$ are uniquely determined by $u\left(x_{0}, t\right)$, and hence also the numbers $c_{k}$ (we may assume $\varphi_{k}\left(x_{0}\right) \neq 0$ or else $\varphi_{k}(x) \equiv 0$ because of (8.4)). Thus

$$
u(x, 1)=\sum_{1}^{\infty} c_{n} \varphi_{n}(x) \exp \left(-\lambda_{n}\right)
$$

is uniquely determined by $u\left(x_{0}, t\right)$. Furthermore, by (8.7),

$$
\begin{aligned}
\|u(x, 1)\|^{\prime \prime} & \leqq \sum_{1}^{\infty}\left|c_{n}\right|\left\|\varphi_{n}(x)\right\| \exp \left(-\operatorname{Re} \lambda_{n}\right) \\
& \leqq B \sum_{1}^{\infty}\left|c_{n}\right|\left|\varphi_{n}\left(x_{0}\right)\right| \exp \left(-\operatorname{Re} \lambda_{n}\right) \\
& \leqq B\left(\sum_{1}^{\infty} \frac{1}{d_{n}} \exp \left(-\operatorname{Re} \lambda_{n}\right)\right)\left\|u\left(x_{0}, t\right)\right\|^{\prime} .
\end{aligned}
$$

The proof is completed by the observation that the last series converges: by (1.9),

$$
\frac{1}{d_{n}} \leqq \exp \left(2 \varepsilon \operatorname{Re} \lambda_{n}\right) \quad \text { as } n \rightarrow \infty
$$

\section{REFERENCES}

1. R. P. Boas, Jr, Entire functions, Academic Press, New York, 1954. MR 16, 914.

2. T. Carleman, Über die Approximation analytischer Funktionen durch lineare Aggregate von vorgegebenen Potenzen, Ark. Mat. Astr. Fys. 17 (1922), no. 9. 
3. M. L. Cartwright, Integral functions, Cambridge Tracts in Math. and Math. Phys., no. 44, Cambridge Univ. Press, New York, 1956. MR 17, 1067.

4. J. A. Clarkson and P. Erdös, Approximation by polynomials, Duke Math. J. 10 (1943), 5-11. MR 4, 196.

5. A. E. Ingham, A note on Fourier transforms, J. London Math. Soc. 9 (1934), 29-32.

6. B. Jessen and A. Wintner, Distribution functions and the Riemann zeta function, Trans. Amer. Math. Soc. 38 (1935), 48-88.

7. T. Kawata, On symmetric Bernoulli convolutions, Amer. J. Math. 62 (1940), 792-794. MR 2, 95.

8. J. Korevaar, $A$ characterization of the sub-manifold of $C[a, b]$ spanned by the sequence $\left\{x^{n} k\right\}$, Nederl. Akad. Wetensch. Proc. Ser. A 50 (1947), 750-758=Indag. Math. 9 (1947), 360-368. MR 9, 346.

9. B. Ja. Levin, Distribution of zeros of entire functions, GITTL, Moscow, 1956; English transl., Transl. Math. Monographs, vol. 5, Amer. Math. Soc., Providence R. I., 1964. MR 19, 402; MR 28 \#217.

10. N. Levinson, On a class of non-vanishing functions, Proc. London Math. Soc. (2) 41 (1936), 393-407.

11. - Gap and density theorems, Amer. Math. Soc. Colloq. Publ., vol. 26, Amer. Math. Soc., Providence, R. I., 1940. MR 2, 180.

12. S. Mandelbrojt, Influence des propriétés arithmétiques des exposants dans une série de Dirichlet, Ann. Sci. École Norm. Sup. (3) 71 (1954), 301-320. MR 16, 815.

13. V. J. Mizel and T. I. Seidman, Observation and prediction for the heat equation, J. Math. Anal. Appl. 28 (1969), 303-312.

14. C. H. Müntz, Über den Approximationssatz von Weierstrass, H. A. Schwarz Festschrift, Berlin, 1914, pp. 303-312.

15. R. E. A. C. Paley and N. Wiener, Fourier transforms in the complex domain, Amer. Math. Soc. Colloq. Publ., vol. 19, Amer. Math. Soc., Providence, R. I., 1934.

16. L. Schwartz, Étude des sommes d'exponentielles réelles, Actualités Sci. Indust., no. 959, Hermann, Paris, 1943. MR 7, 294.

17. O. Szász, Über die Approximation stetiger Funktionen durch lineare Aggregate von Potenzen, Math. Ann. 77 (1915), 482-496.

18. A. Wintner, On analytic convolutions of Bernoulli distributions, Amer. J. Math. 56 (1934), 659-663.

19. - On symmetric Bernoulli convolutions, Bull. Amer. Math. Soc. 41 (1935), 137-138.

20. - On convergent Poisson convolutions, Amer. J. Math. 57 (1935), 827-838.

21. - On a class of Fourier transforms, Amer. J. Math. 58 (1936), 45-90.

\section{California Institute of Technology, Pasadena, California 91109 \\ UNIVERSITY OF CALIFORNIA, San Diego, California 92037}

